ARTIGO ORIGINAL

\title{
Efeito da atividade condicionante com diferentes intensidades sobre o desempenho do salto vertical
}

\author{
Effect of conditioning contraction with different intensities on vertical jump performance \\ Danilo Jamaikon Carvalho ${ }^{1}$, Heitor Francisco Moreira Barbosa ${ }^{1}$, Ramon Franco Carvalho ${ }^{1,2}$ \\ ${ }^{1}$ Centro Universitário Presidente Antônio Carlos (UNIPAC), Barbacena/MG, Brasil \\ 2 Universidade do Estado do Rio de Janeiro (UERJ), Rio de Janeiro/RJ, Brasil
}

\author{
HISTÓRICO DO ARTIGO \\ Recebido: 02 maio 2020 \\ $1^{\text {a. }}$ Revisão: 28 junho 2020 \\ 2. Revisão: 28 outubro 2020 \\ Aprovado: 03 novembro 2020
}

\section{PALAVRAS-CHAVE:}

Força muscular; Fadiga;

Contração muscular.

\section{KEYWORDS:}

Muscle strength; Fatigue;

Muscular contraction.

\section{RESUMO}

INTRODUÇÃo: A potencialização pós ativação é o fenômeno do aumento da potência muscular quando precedido de uma atividade condicionante $(A C)$ de força a partir de cargas de alta intensidade.

OBJETIVO: Verificar o efeito agudo de três diferentes intensidades de atividade condicionante sobre o desempenho do salto vertical.

MÉTODOS: Quinze homens com experiência no treinamento de força visitaram o laboratório em seis ocasiões. As duas primeiras visitas foram destinadas exclusivamente para identificar a carga de 1RM no exercício agachamento. As demais visitas foram realizadas de forma aleatória compreendendo três condições experimentais e um controle. Os procedimentos experimentais foram compostos com a AC realizada em 3 séries de 3 repetições com 20,55 ou 90\% de 1RM no exercício agachamento. Tanto antes, como 10 minutos após a realização da AC, os voluntários realizaram 3 saltos verticais. Na visita controle, nenhuma AC foi realizada entre as séries de saltos. Como variável dependente, tanto a maior altura obtida (melhor desempenho) quanto a média de altura das séries de saltos foram consideradas. Após a verificação da normalidade pelo teste de Shapiro-Wilk, uma ANOVA two-way com medidas repetidas foi utilizado para verificar se havia interação ou efeito principal. $O$ nível de significância adotado foi de $p<0,05$.

RESULTADOS: ANOVA two-way com medidas repetidas não observou interação grupo $x$ teste $(p=0,938)$ e nem efeito principal grupo $(p=0,486)$ ou teste $(p=0,658)$ quando se considerou a média dos 3 saltos. 0 mesmo resultado foi observado quando se avaliou o salto de melhor desempenho ( $p=0,582 ; p=0,873 ; p=0,119)$.

CONCLUSÃO: Os protocolos adotados no presente estudo parecem não interferir no desempenho do salto vertical para indivíduos experientes no treinamento de força, mas não atletas. A individualidade biológica é um importante fator de influência, indicando a necessidade de testar o desempenho individual antes de prescrever este modelo de treino.

\section{ABSTRACT}

BACKGROUND: Post-activation potentiation is the phenomenon of increased muscle power when preceded by a high intensity conditioning activity (CA).

OBJECTIVE: To verify the acute effect of three different intensities of conditioning activity on the vertical jump performance.

METHODS: Fifteen men with strength training experience visited the laboratory on six occasions. The first two visits were intended exclusively to identify the 1 RM load in the squat exercise. The other visits were carried out at random, comprising three experimental conditions and one control. The experimental procedures were composed with the CA performed in 3 sets of 3 repetitions with 20,55 or $90 \%$ of 1 RM in the squat exercise. Before and 10 minutes after performing the CA, the volunteers performed 3 vertical jumps. In the control visit, no CA was performed between the sets of jumps. As a dependent variable, both the highest height obtained (best performance) and the average height of each sets of jumps were considered. After checking the normality by the Shapiro-Wilk test, a two-way ANOVA with repeated measures was used to check if there was an interaction or main effect. The level of significance was $p<0.05$.

RESULTS: Two-way ANOVA with repeated measures did not observe group $x$ test interaction $(p=0.938)$ and neither main group effect $(p=0.486)$ nor test $(p=0.658)$ when considering the average of the 3 jumps. The same result was observed when the best performance jump was evaluated $(p=0.582 ; p=0.873 ; p=0.119)$.

CONCLUSION: The protocols adopted in the present study do not seem to interfere with the vertical jump performance for individuals experienced in strength training, but not athletes. Biological individuality is an important influencing factor, indicating the need to test individual performance before prescribing this training model. 


\section{INTRODUÇÃO}

O treinamento complexo pode ser caracterizado como um modelo de treino constituído pela combinação de exercícios de força máxima ou próxima da máxima seguido por atividades de característica de força explosiva cujo objetivo é promover a melhora da potência muscular. Esse fenômeno do aumento agudo da potência muscular é conhecido como potencialização pós ativação (PPA), enquanto o exercício de força é chamado de atividade condicionante (AC) (HODGSON; DOCHERTY; ROBBINS, 2005; SALE, 2002; TILLIN; BISHOP, 2009). Os mecanismos fisiológicos que explicam o efeito potencializador são a fosforilação da cadeia leve regulatória da miosina (CRLM) que aumenta a sensibilidade da troponina com o cálcio liberado pelo retículo sarcoplasmático (MOORE; STULL, 1984; SZCZESNA et al., 2002; VANDENBOOM; GRANGE; HOUSTON, 1995), um possível maior recrutamento de unidades motoras de alto limiar de excitabilidade (LUSCHER; RUENZEL; HENNEMAN, 1983), além de uma controversa redução aguda no ângulo de penação (MAHLFELD; FRANKE; AWISZUS, 2004).

A observação do fenômeno da PPA depende da relação de vários fatores, como a característica da população (HAMADA; SALE; MacDOUGALL, 2000; PÄÄSUKE et al., 2007; RIXON; LAMONT; BEMBEN, 2007), o intervalo entre $A C$ e atividade principal (GILBERT; LEES, 2005; GOUVÊA et al., 2013), o volume (FRENCH; KRAEMER; COOKE, 2003), a intensidade da AC (COMYNS et al., 2007; HANSON; LEIGH; MYNARK, 2007; ROBBINS; DOCHERTY, 2005) e tipos de fibras musculares (HAMADA; SALE; MacDOUGALL, 2000; SZCZESNA et al., 2002). Considerando a variável intensidade, esta parece influenciar significativamente no fenômeno da PPA, pois há uma relação direta com o recrutamento de unidade motoras do tipo II (HAMADA; SALE; MacDOUGALL, 2000). Com isso alguns autores recomendam que a $A C$ deve ser realizada com uma sobrecarga máxima ou próxima da máxima (SALE, 2002; TILLIN; BISHOP, 2009). Todavia, a velocidade também pode ser um fator de recrutamento de unidades motoras do tipo II. Assim, em teoria, o fenômeno da PPA pode ser estimulado pela AC realizada tanto através da mobilização de uma elevada carga resistiva quanto através de sua execução em alta velocidade (HARRISON et al., 2019).

Alguns trabalhos buscaram compreender o efeito de diferentes intensidades no agachamento, utilizado como AC, sobre o desempenho do salto vertical subsequente (COMYNS et al., 2007; GILBERT; LEES, 2005; HANSON; LEIGH; MYNARK, 2007). Apenas o estudo de Hanson et al. (2007) utilizou uma sobrecarga no agachamento relativamente baixa (8 repetições com $40 \%$ da força máxima obtida no teste de uma repetição máxima 1RM) em comparação a uma carga relativamente alta (4 repetições com $80 \%$ de $1 \mathrm{RM}$ ), porém com a velocidade controlada por metrônomo. Apesar do mérito dos autores em buscar igualar o estresse metabólico e neuromuscular para separar apenas a influência da intensidade da carga do agachamento, o controle da velocidade de execução provavelmente causou um viés. O percentual da carga máxima representa uma larga margem de repetições máximas possíveis de serem executadas, principalmente em exercício de membro inferior e multi-articular (HOEGER et al., 1990). Com isso dobrar o número de repetições de um exercício a ser realizado com metade da carga relativa (percentual de 1RM) não representa necessariamente o mesmo estresse metabólico ou neuromuscular. Assim a execução em velocidade máxima livre, mesmo não sendo capaz de igualar, pode ao menos minimizar essa diferença.

Além disso, a literatura ainda é controversa quanto aos possíveis efeitos benéficos sobre o desempenho neuromuscular em sujeitos ativos submetidos a este método de treino (HAMADA; SALE; MacDOUGALL, 2000; KHAMOUI et al., 2009). Uma possível explicação pode ser a característica da AC utilizada em ambos os estudos. O efeito da PPA foi observado nessa população quando a AC foi realizada em esforço máximo isométrico. Todavia quando realizado com agachamento dinâmico a $85 \%$ de $1 R M$, nenhum efeito potencializador ocorreu indicando que a fadiga pode ter contrabalanceado os mecanismos da PPA. No entanto, não se sabe se uma menor intensidade relativa na AC poderia minimizar os efeitos da fadiga e estimular a predominância da PPA. Sendo assim, o presente estudo visa verificar o efeito da AC realizada em diferentes percentuais de carga de $1 R M$ no desempenho do salto vertical em homens ativos e treinados em força, mas não atletas.

\section{MÉTOdOS}

\section{Participantes}

Participaram do presente estudo 15 homens saudáveis (24,9 $\pm 5,9$ anos; $174,3 \pm 6,3 \mathrm{~cm} ; 76,5 \pm 8,8 \mathrm{~kg} ; 16,0 \pm 5,7 \% \mathrm{HC}$; carga de $1 \mathrm{RM}=103,1 \pm 20,3 \mathrm{~kg}$ ), com experiência em treinamento de força. Como critério de inclusão do estudo, os participantes deveriam ser treinados em força a mais de um ano, com frequência semanal mínima de 3 vezes e que fossem capazes de mobilizar uma carga igual ou superior a sua massa corporal no exercício agachamento durante o teste de carga máxima equivalente a uma repetição máxima (1RM). Por outro lado, foram excluídos da amostra todos os indivíduos que apresentaram diagnóstico de lesão osteomioarticular ou relataram dor que impossibilitasse a realização de qualquer exercício que fizesse parte do protocolo deste estudo, além dos que relataram uso de esteroides anabolizantes nos 12 meses que antecederam esta pesquisa.

Antes de iniciar a participação como voluntários, todos receberam informações sobre o estudo e assinaram o Termo de Consentimento Livre e Esclarecido (TCLE). O estudo foi aprovado pelo Comitê de Ética em Pesquisa da Universidade Presidente Antônio Carlos (UNIPAC), com o Parecer $n^{\circ}$. 1.916.007.

\section{Delineamento da pesquisa}

Os participantes visitaram uma academia na cidade do Alto do Rio Doce (MG) em seis ocasiões. O intervalo entre as visitas foi de no mínimo dois dias e máximo de uma semana. As duas primeiras visitas foram destinadas a identificação de 1RM no exercício agachamento. Posteriormente, essas medidas foram utilizadas para verificar a confiabilidade da carga que serviu de base para a escolha das intensidades utilizadas no presente estudo. As últimas quatro visitas, destinadas para a execução dos três procedimentos experimentais e um controle, foram realizadas de forma aleatória entre os participantes. A sequência foi definida através de um sorteio realizado ao final da segunda visita.

Nas visitas referentes aos tratamentos experimentais, inicialmente os sujeitos foram submetidos a uma série de três sal- 
tos verticais do tipo contra movimento com intervalo de $15 \mathrm{se}$ gundos entre eles. Tanto o salto de melhor desempenho quanto a média entre os três, foram obtidas como medidas de baseline. Após um intervalo de cinco minutos, os participantes foram submetidos a um dos protocolos do teste experimental, com a execução do exercício agachamento no aparelho Smith em três séries de três repetições com o máximo de velocidade possível. $O$ intervalo entre as séries de agachamento foi de três minutos.

O agachamento foi escolhido como AC devido sua cinemática permitir a estimulação dos mesmos grupamentos musculares envolvidos no salto vertical, que foi o exercício adotado para obter a variável dependente. As cargas da AC utilizadas nas visitas experimentais foram equivalentes a 20,55 ou $90 \%$ de 1 RM. Após o intervalo de 10 minutos, os participantes repetiram o mesmo procedimento do teste de salto realizado na condição pré-tratamento.

A amplitude no agachamento foi controlada pela angulação dos joelhos. A medida foi obtida na primeira visita, antes de realizar o teste de carga máxima, utilizando um goniômetro com o sujeito em isometria. Após atingir a angulação de $90^{\circ}$ no joelho, uma trava móvel foi colocada no equipamento para limitar o movimento do participante a esta amplitude articular. Além de indicar a amplitude que o voluntário deveria realizar em todos os movimentos e evitar uma possível variável interveniente causada por diferentes amplitudes, a trava também serviu como uma medida de segurança aos participantes em caso de falha na execução em alguma repetição por fadiga.

$\mathrm{Na}$ visita controle, o sujeito realizou o mesmo procedimento descrito nas condições experimentais, porém sem a realização do agachamento na AC. Assim, somente as séries de testes de saltos pré e pós foram realizados com intervalo de $22 \mathrm{mi}$ nutos entre elas, para respeitar o mesmo intervalo observado nas condições experimentais. Para evitar um possível viés que comprometesse o resultado das variáveis dependentes, nenhuma atividade de aquecimento foi realizada nos protocolos experimentais e controle. A Figura 1 ilustra os procedimentos experimentais e controle descritos acima que foram realizados de forma aleatória.

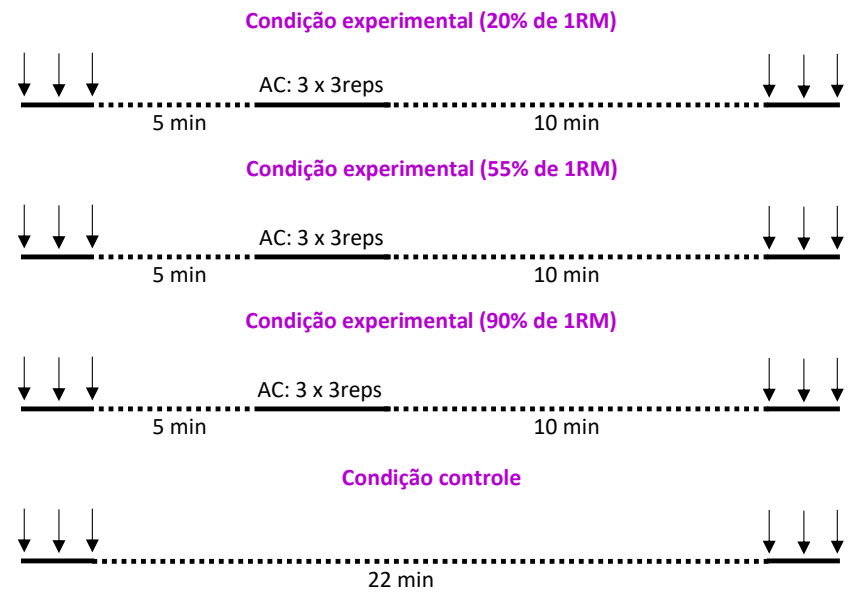

Figura 1. Design da pesquisa com as condições experimentais e controle realizadas de forma aleatória.

Legenda: Seta indica o momento de realização de cada salto vertical. 1RM significa a carga equivalente a uma repetição máxima. AC: Atividade Condicionante; reps: repetições; min: minutos
Para evitar qualquer efeito deletério de atividades prévias, os participantes foram informados a não participar de qualquer exercício de flexibilidade, força ou resistência de membros inferiores até $48 \mathrm{~h}$ antes dos testes.

\section{Descrição do Agachamento no Smith (Atividade Condicionante)}

A barra do aparelho Smith (Movement, modelo convencional, Pompéia, São Paulo, Brasil) foi apoiada sobre a parte superior das escápulas, aproximadamente na altura da segunda ou terceira vértebra torácica. As mãos do sujeito ficaram apoiadas na barra na posição de maior conforto, e a distância entre os pés seguiu o mesmo critério. Foi considerado como posição inicial o sujeito em pé com o tronco no mesmo plano frontal dos membros inferiores. A fase excêntrica do movimento foi realizada com a flexão do quadril, joelhos e tornozelos até a barra encostar na trava do aparelho. Assim que finalizava a fase excêntrica, o sujeito deveria realizar imediatamente o movimento concêntrico na maior velocidade possível até retornar à posição inicial. Todas as repetições alcançaram todo o arco de movimento pré-estabelecido pelo protocolo e foram executadas sem auxílio externo para concluir qualquer uma das fases de contração descritas.

\section{Teste de carga máxima no agachamento}

Na primeira visita, os sujeitos indicaram a carga que acreditavam corresponder a $1 \mathrm{RM}$ no exercício agachamento realizado no aparelho Smith. O teste foi iniciado com $90 \%$ da carga estimada pelo sujeito. Conseguindo realizar uma repetição sem auxílio externo, a execução era repetida após cinco minutos de intervalo e com uma carga adicional de $10 \mathrm{~kg}$. Esse procedimento foi repetido tantas quantas vezes necessário. Quando o sujeito falhou na tentativa de mobilizar a carga, mais uma única tentativa foi oportunizada com a subtração de $5 \mathrm{~kg}$. Foi considerada como carga referente à $1 \mathrm{RM}$ aquela correspondente a realizada na última execução bem-sucedida.

Para o reteste da carga de 1 RM (segunda visita), o sujeito iniciou com a carga obtida no primeiro teste. Todos os procedimentos descritos anteriormente de incremento e subtração de carga foram repetidos. Para o reteste, todos os sujeitos atingiram a carga máxima em menos de 5 tentativas. Somente foram consideradas válidas as repetições que percorreram todo o arco de movimento, previamente definida. Durante a realização do teste no agachamento, ao menos dois pesquisadores acompanharam todo o procedimento para garantir a segurança dos avaliados. Os procedimentos adotados nos testes de carga equivalente a 1RM seguiram a recomendação da Sociedade Americana dos Fisiologistas do Exercício (BROWN; WEIR, 2001).

\section{Descrição do Salto Vertical}

Para realizar o salto vertical, o voluntário posicionou-se de pé com o peso distribuído em ambos os membros inferiores. A distância entre os pés foi a distância de maior conforto para cada sujeito, o pé direito ficou sobre uma fita vermelha fixada no chão que serviu de marcação para delimitar o espaço para a execução do SV. Os sujeitos se posicionaram com o lado direito voltado para a câmera e foi realizada uma marcação em cima do maléolo lateral para melhor visualização. Ao comando do 
avaliador, o sujeito realizou um rápido movimento de flexão de quadril e joelhos sendo sucedido por um vigoroso movimento de extensão dessas articulações (ciclo alongamento encurtamento). Para a validação do salto foi necessário obedecer aos seguintes critérios: a) Em todos os momentos de cada salto o avaliado permaneceu com as mãos na cintura; b) Ao atingir o solo os joelhos deveriam estar em extensão; c) Não poderia haver deslocamento do local de voo para o de aterrissagem além das marcações que estavam no solo (fitas vermelhas); d) O sujeito não poderia saltar antes da liberação sonora concedida pelo avaliador.

\section{Descrição do procedimento de fotogrametria}

A altura do salto vertical foi obtida através da análise das imagens pelo método de videogrametria. Essa técnica de mensuração do salto vertical foi validada por Moura et al. (2015). Essa avaliação constituiu na utilização de uma câmera de vídeo Samsung, modelo PL20, de 14,2 megapixels, com alcance de zoom óptico de $5 x$ e com frequência de $24 \mathrm{~Hz}$, para coletar as imagens do salto vertical. Após o registro dos saltos, as imagens foram passadas para um computador. Com a utilização do programa QuickTime Player $^{\circledR}$ foi possível desmembrar a filmagem em quadro a quadro, e assim foi observado o ponto mais alto em que os participantes saltaram. Em seguida foi utilizado o programa OneNote 2013 (Pacote Microsoft Office 2013 ${ }^{\circledR}$ ) para extrair as imagens do vídeo, que foi posteriormente analisado através do programa ImageJ para obtenção da medida da altura do salto.

O posicionamento da câmera se deu a um metro e meio do local onde o sujeito realizou o salto e com a altura da lente em relação ao solo de $20 \mathrm{~cm}$. Para obter a medida da altura alcança$\mathrm{da}$, antes de saltar os sujeitos se posicionaram como lado direito voltado para a câmera e foi realizada uma marcação sobre o maléolo lateral. Uma fita adesiva vermelha foi fixada no solo, ao lado do pé direito e um gabarito de altura conhecida $(70 \mathrm{~cm})$ foi posicionado ao lado dos sujeitos. Após autorização do pesquisador, os sujeitos realizaram o salto na maior intensidade possível enquanto todo o movimento era filmado. Para a obtenção da medida da altura do salto vertical pelo programa ImageJ, uma linha no programa foi traçada entre a marcação do maléolo até a fita vermelha no solo utilizando o gabarito como referência de altura. Após o resultado informado no programa, a medida da altura do maléolo em relação ao solo obtida antes realizar o salto vertical foi subtraída para alcançar o resultado final da medida correspondente à altura do salto.

\section{Análise estatística}

O teste de Shapiro-Wilk foi utilizado para verificar o pressuposto de normalidade dos dados. Todos os valores foram reportados em média e desvio padrão. Para determinar a qualidade e confiabilidade das medidas de carga equivalente a $1 \mathrm{RM}$ e nos testes de salto vertical foram verificados o erro típico da medida de acordo com a recomendação de Hopkins (HOPKINS, 2000), o Coeficiente de Correlação Intraclasse e a análise gráfica de Bland-Altman. Uma ANOVA two-way com medidas repetidas nos dois fatores ( $4 \times 2$; grupos e testes) foi calculada para determinar se houve interação grupo $x$ testes ou efeito principal. 0 nível de significância adotado no presente estudo foi $p<0,05$. Além disso, o tamanho do efeito foi calculado para identificar a magnitude da diferença. Valores menores que 0,35 foram considerados como trivial, aqueles entre 0,35 a 0,80 como pequeno, de 0,81 a 1,50 como moderado e acima de 1,50 como alto para indivíduos treinados recreacionalmente (RHEA, 2004). Todas as análises foram realizadas pelo pacote de programa estatístico SPSS 21.0 (SPSS Inc., EUA).

\section{RESULTADOS}

O Coeficiente de Correlação Intraclasse indicou alta correlação entre os saltos verticais considerando tanto a média quanto o melhor desempenho. O mesmo pode se observar nos testes de 1 RM. O baixo Erro Típico da Medida das variáveis dependentes (altura dos saltos) e da carga equivalente a 1RM também reforçou a qualidade dos dados medidos. Esses resultados estão apresentados na Tabela 1.

Tabela 1. Resultado do Coeficiente de Correlação Intraclasse (CCI) e Erro Típico da Medida (ETM) para o teste de força máxima e salto verticais.

\begin{tabular}{lcccc}
\hline Medidas & $\mathbf{C C l}$ & $\mathbf{p}$ & ETM (abs) & ETM (rel) \\
\hline Teste de 1RM & 0,97 & 0,000 & $5,92 \mathrm{~kg}$ & $5,92 \%$ \\
SV média & 0,99 & 0,000 & $0,64 \mathrm{~cm}$ & $1,48 \%$ \\
SV pico & 0,98 & 0,000 & $1,01 \mathrm{~cm}$ & $2,28 \%$ \\
\hline
\end{tabular}

A análise gráfica de Bland-Altman também demonstrou que os desempenhos dos saltos possuem alta confiabilidade com todos os participantes estando dentro do limite de concordância de 1,96 DP. No entanto, o gráfico apresentou uma tendência de erro heterocedástico considerando a maior altura obtida no salto vertical (salto de melhor desempenho). As Figuras 2 e 3 apresentam a análise gráfica de Bland-Altman respectivamente para as variáveis dependentes (média e salto de melhor desempenho) e para a carga máxima equivalente a 1RM.
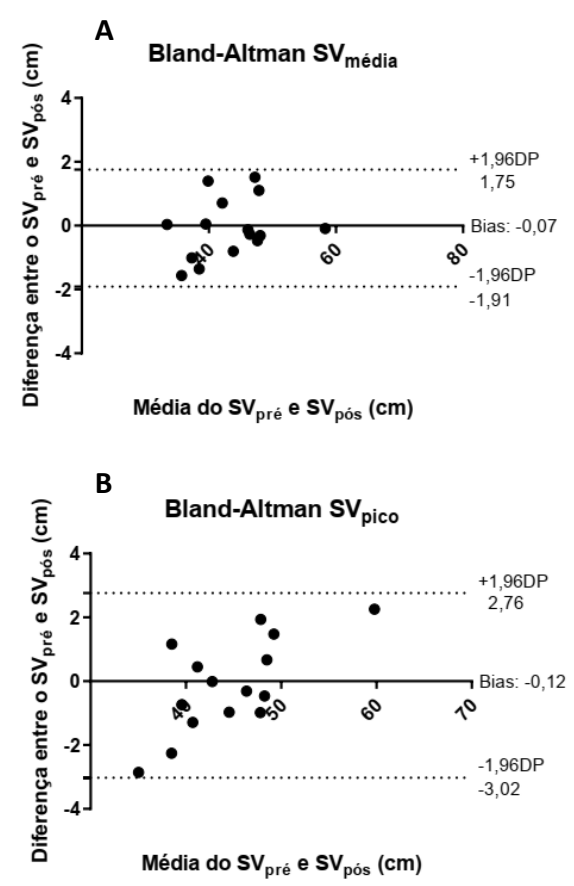

Figura 2. Limites de concordância entre (2A) os valores médios de salto vertical máximo $(\mathrm{cm})$ comparadas em pares (teste pré vs. teste pós na condição controle) considerando a diferença e a média entre os SV e (2B) os valores máximos de salto vertical máximo $(\mathrm{cm})$ comparadas em pares (teste pré vs. teste pós na condição controle) considerando a diferença e a média entre os SV. 


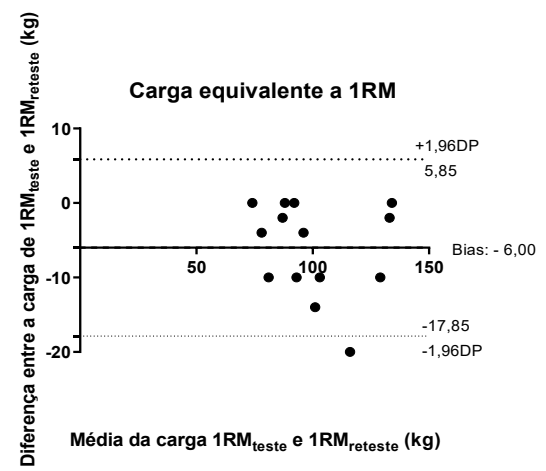

Figura 3. Limites de concordância entre os valores de carga máxima equivalente a $1 \mathrm{RM}(\mathrm{kg})$ comparadas em pares (teste vs. reteste) considerando a diferença e a média entre as cargas máximas

A ANOVA two-way com medidas repetidas nos dois fatores não observou diferença significativa entre os grupos $(p=0,486)$, testes $(p=0,658)$ e nem interação entre grupo e teste $(p=0,938)$ quando se considerou a média dos saltos realizados na condição pré e pós e entre as distintas condições experimentais e controle. Resultado semelhante ocorreu grupo $(p=0,873)$, teste $(p=0,119)$ e interação grupo e teste $(p=0,582)$ quando a análise considerou apenas o salto de melhor desempenho. A Figura 4 apresenta os resultados em média e desvio padrão do desempenho no salto vertical e a Figura 5 apresenta os resultados individuais de cada participante.

A

Média dos três SV

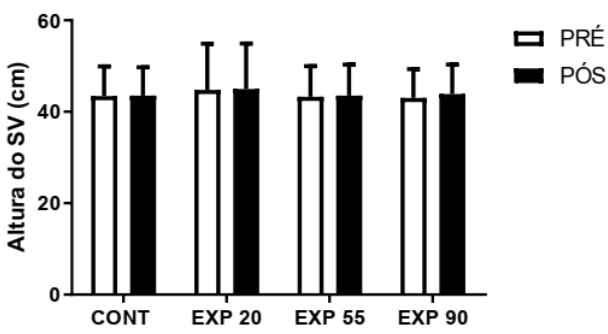

B

Melhor desempenho do SV

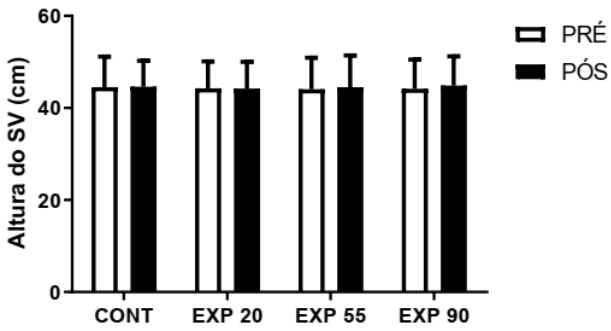

Figura 4. Valores de impulsão vertical do salto contra movimento. Os valores são apresentados em média e desvio padrão. Barra clara indica os valores das medidas pré e barra escura para as medidas pós. A Figura 4A apresenta o resultado considerando a média dos 3 saltos e a 4B apresenta o resultado considerando apenas o salto de melhor desempenho.

Legendas: SV: salto vertical. CONT: Condição Controle. EXP20: Condição Experimental com AC a 20\% de uma repetição máxima. EXP55: Condição Experimental com AC a 55\% de uma repetição máxima. EXP90: Condiçã̃o Experimental com AC a 90\% de uma repetição máxima.
A

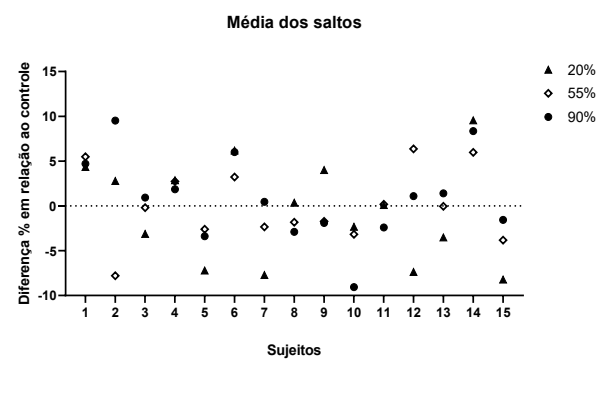

B

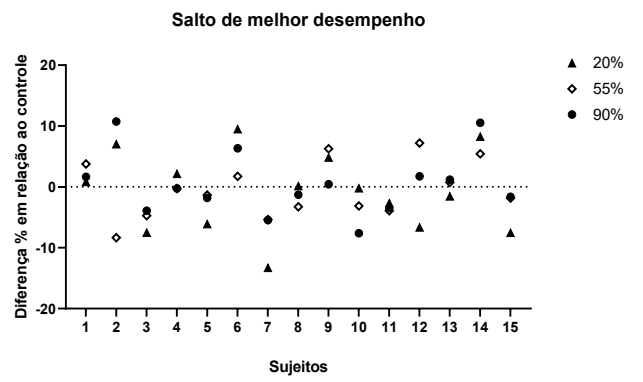

Figura 5. Variabilidade dos resultados individuais no desempenho do salto vertical. A Figura $5 \mathrm{~A}$ apresenta a variação percentual das médias dos saltos verticais em relação ao controle quando a atividade condicionante foi realizada com $20 \%(\Delta), 55 \%(\diamond)$ e $90 \%(\bullet)$ de uma repetição máxima. A Figura $5 \mathrm{~B}$ apresenta a variação percentual do salto vertical de melhor desempenho em relação ao controle quando a atividade condicionante foi realizada com $20 \%(\Delta) 55 \%(\diamond)$ e $90 \%(\bullet)$ de uma repetição máxima.

O tamanho do efeito para os valores da média dos saltos variou de 0,01 a 0,13. Resultado semelhante se observou quando considerou apenas os saltos de melhor desempenho, com o tamanho do efeito variando de 0,01 a 0,11. Em todas essas condições o tamanho do efeito foi classificado de trivial. Os resultados estão apresentados nas Tabelas 2.

Tabela 2. Tamanho do efeito da média de altura obtida no salto vertical e altura máxima no salto vertical, precedido pela atividade condicionante em diferentes intensidades comparado as medidas de baseline (pós vs. pré).

\begin{tabular}{cccc}
\hline \multirow{2}{*}{ Tamanho do Efeito } & \multicolumn{3}{c}{ Intensidade da atividade condicionante } \\
\cline { 2 - 4 } & $\mathbf{2 0 \%}$ & $\mathbf{5 5 \%}$ & $\mathbf{9 0 \%}$ \\
\hline Média de altura do salto vertical & 0,03 & 0,04 & 0,13 \\
Altura máxima do salto vertical & $-0,01$ & 0,07 & 0,11 \\
\hline
\end{tabular}

A escolha do salto como atividade principal a ser potencializada pela AC foi devido a sua presença em diversas modalidades desportivas e capacidade preditiva de desempenho de potência muscular (ALMUZAINI; FLECK, 2008). Além disso, estudo prévio já demonstrou que o salto vertical possui alta confiabilidade em uma população semelhante ao presente estudo (CARVALHO; RUBINI; CABRAL, 2014). No entanto, o presente estudo não observou predominância de desempenho no salto vertical quando precedida por uma AC de elevada carga resistiva quando comparada a menores cargas ou mesmo com a situação controle. Como a literatura já demonstrou haver uma coexistência entre os mecanismos de fadiga e PPA (RASSIER; MACINTOSH, 2000), algumas possíveis explicações podem ser especuladas. A recomendação para a realização da $A C$ na maior velocidade de 
movimento possível, incluindo quando executado com apenas $20 \%$ de $1 \mathrm{RM}$, pode estimulado igualmente os mecanismos da PPA e da fadiga, assim como a realização da AC com cargas mais elevadas. Porém, uma das limitações do presente estudo foi a ausência de mensuração de sinais eletromiográficos para confirmar essa hipótese.

Outra possível causa pode estar associada ao intervalo de 10 minutos de recuperação adotado no presente estudo. Esse intervalo foi escolhido devido a pesquisas prévias que indicaram que esta seria a "janela ótima" para se observar a PPA durante a atividade principal realizada com exercício com ciclo alongamento e encurtamento, característico de atividades de força explosiva como o salto vertical (GOUVÊA et al., 2013; KILDUFF et al., 2007). A meta-análise realizada por Gouvêa et al. (2013) demonstrou que o intervalo entre 8 a 12 minutos parece ser o ideal para que os mecanismos da PPA sobressaiam sobre a fadiga, obtendo assim o melhor desempenho no salto vertical (GOUVÊA et al., 2013). Tal resultado foi suportado por Kilduff et al. (2007) que também encontraram o mesmo intervalo como sendo ideal para o desempenho do arremesso após AC. Apesar do presente estudo ter avaliado o desempenho do salto dentro do período de recuperação recomendado pela literatura, não foi capaz de observar predominância da PPA ou da fadiga no desempenho do salto, indicando um equilíbrio entre os mecanismos associados por ambos os fenômenos.

Gilbert e Lee (2005) observaram que AC realizada com alta intensidade baseada na carga, assim como a realizada com a carga que estimulava a maior potência muscular foram efetivas para se observar a PPA. No entanto, o comportamento do desempenho alterou de acordo com a característica da AC. Em ambas as condições, a AC foi realizada no exercício agachamento em 5 séries de 1 repetição e intervalo entre as séries de $5 \mathrm{mi}$ nutos. Porém, em uma das condições experimentais os sujeitos executaram com a carga equivalente a $1 \mathrm{RM}$ enquanto na outra condição a carga foi submáxima, mas que equivalia a carga que gerava maior potência muscular considerando a relação força e velocidade. Para isso, todos os sujeitos tiveram que mobilizar as cargas de $\mathrm{AC}$ na maior velocidade possível. Na condição com carga máxima na $\mathrm{AC}$, os sujeitos alcançaram o melhor desempenho no salto vertical com 20 minutos de intervalo de recuperação enquanto na condição da carga submáxima que gerava maior potência, os sujeitos alcançaram o melhor desempenho com apenas 2 minutos de recuperação. Ao comparar com presente estudo que adotou um intervalo único de recuperação (10 minutos) com os resultados observados por Gilbert e Lee (2005), é possível estimar que o efeito potencializador estimulados nas condições de 20 e $55 \%$ de 1 RM já tenham sido dissipados, enquanto na carga próxima a máxima ( $90 \%$ de $1 \mathrm{RM}$ ) ainda estaria em progressão.

A literatura científica já demonstrou que sujeitos treinados em força podem se beneficiar do treinamento complexo (HAMADA; SALE; MacDOUGALL, 2000). No entanto, o presente estudo não foi capaz de confirmar este achado. De fato, atletas são mais suscetíveis aos efeitos benéficos deste modelo de treino pelo maior nível de treinamento, o que pode contribuir a uma maior resistência a fadiga ou mais sensíveis aos mecanismos da PPA (HAMADA; SALE; MacDOUGALL, 2000; PÄÄSUKE et al., 2007; RIXON; LAMONT; BEMBEN, 2007). Isto contribuiu para explicar a diferença em relação a outro estudo que observou melhora do desempenho no salto profundo unilateral realizado por atletas de Rugby num trenó e partindo de uma altura prévia de $30 \mathrm{~cm}$ (COMYNS et al., 2007). Neste estudo, os autores compararam o desempenho em diversas variáveis que afetam a altura do salto quando precedidas por AC de diferentes intensidades (65, 80 e 93\% de 1RM). Todas as condições de carga produziram melhora no tempo de voo (variável que afeta diretamente a altura do salto realizado pelos sujeitos) em relação ao controle, mas somente a carga de $93 \%$ de 1RM proporcionou aumento na rigidez da perna e redução no tempo de contato.

Ao observar as respostas individuais dos sujeitos foi identificado uma variabilidade (individualidade) nas respostas em relação ao estímulo adotado no presente estudo. Como observado na Figura 5, o mesmo indivíduo poderia experimentar tanto a melhora quanto a piora na altura do salto dependendo da condição experimental a que foi submetido. Assim, nove dos voluntários conseguiram melhorar a média dos saltos verticais e 11 pioraram em alguma condição experimental a que foram submetidos. A mesma característica se observou quando a variável dependente foi o melhor salto obtido, com sete experimentando melhora individual em relação ao controle em alguma condição experimental e 10 deles pioram o desempenho. Essa variabilidade nas respostas ao estímulo condicionante, promovida pela coexistência entre os mecanismos de PPA e fadiga, também foi observado em estudos prévios (BEVAN et al., 2010; JO et al., 2010). De certa forma, essa grande variabilidade observada nos estudos de treinamento complexo pode ser explicada por fatores intrínsecos relacionados as características dos sujeitos, como a predominância do tipo de fibras e o nível de treinamento, assim como por fatores extrínsecos relacionados a combinação das variáveis de treino, como intervalo, intensidade e volume. Assim existe a necessidade de testar a eficiência individual de cada sujeito que pretenda incluir este método (treinamento complexo) em seu programa de treino.

\section{CONCLUSÃO}

O presente estudo não observou alteração no desempenho do salto realizado por homens ativos em treinamento de força (mas não atletas) após a AC realizada com diferentes intensidades de carga (20, 55 e $90 \%$ de $1 \mathrm{RM}$ ) e dentro do intervalo de recuperação adotado. Apesar da literatura suportar a aplicação deste método para atletas de modalidades de predominância de força ou potência muscular, ainda não é clara sua efetividade em população similar ao do presente estudo.

Apesar de aparentemente não interferir significativamente para a maioria dos indivíduos ativos não atletas, a individualidade biológica observada indica a necessidade de aprofundamento do conhecimento com novas pesquisas para observar como a manipulação das variáveis de treinamento (volume, intensidade, intervalo, entre outras) podem interferir nesse comportamento. Além disso também demonstra a necessidade de se testar previamente as diferentes combinações de carga antes incluir este modelo num programa de treinamento para obter uma prescrição individualizada e efetiva.

\section{REFERÊNCIAS}

ALMUZAINI, K. S; FLECK, S. J. Modification of the standing long jump test enhances ability to predict anaerobic performance. Strength and Conditioning Journal, Philadelphia, v. 22, p. 1265-72, 2008. 
BEVAN, H. R.; CUNNINGHAM, D. J.; TOOLEY, E. P.; OWEN, N. J.; COOK, C. J.; KILDUFF, L. P. Influence of postactivation potentiation on sprinting performance in professional rugby players. Journal of Strength and Conditioning Research, Philadelphia, v. 24, n. 3, p. 701-5, 2010

BROWN, L. E.; WEIR, J. P. ASEP procedures recommendation I: Accurate assessment of muscular strength and power. Journal of Exercise Physiology Online, Beaumont, v. 4, n. 11, p. 1-21, 2001

CARVALHO, R. F.; RUBINI, E. C.; CABRAL, L. F. Estabilidade no desempenho de três diferentes técnicas de salto vertical. Caderno de Educação Física e Esporte, Marechal Cândido Rondon, v. 12, n. 2, p. 21-9, 2014

COMYNS, T. M.; HARRISON, A. J.; HENNESSY, L.; JENSEN, R. L. Identifying the optimal resistive load for complex training in male rugby players. Sports Biomechanics, Abingdon, v. 6, n. 1, p. 59-70, 2007.

FRENCH, D. N.; KRAEMER, W. J.; COOKE, C. B. Changes in Dynamic Exercise Performance Following a Sequence of Preconditioning Isometric Muscle Actions. Journal of Strength and Conditioning Research, Philadelphia, v. 17, n. 4 , p. $678-85,2003$

GILBERT, G.; LEES, A. Changes in the force development characteristics of muscle following repeated maximum force and power exercise. Ergonomics, Abingdon, v. 48, n. 11-14, p. 1576-84, 2005.

GOUVÊA, A. L.; FERNANDES, I. A.; CÉSAR, E. P.; SILVA, W. A. B.; GOMES, P. S. $C$. The effects of rest intervals on jumping performance: A meta-analysis on post-activation potentiation studies. Journal of Sports Sciences, London, v. 31, n. 5, p. $459-467,2013$.

HAMADA, T.; SALE, D. G.; MacDOUGALL, J. D. Postactivation potentiation in endurance-trained male athletes. Medicine and Science in Sports and Exercise, Indianapolis, v. 32, n. 3, p. 403-11, 2000

HANSON, E. D.; LEIGH, S.; MYNARK, R. G. Acute effects of heavy-and ligh$\mathrm{t}$-load squat exercise on the kinetic measures of vertical jumping. Journal of Strength and Conditioning Research, Philadelphia, v. 21, n. 4, p. 1012-7, 2007

HARRISON, P. W.; JAMES, L. P.; McGUIGAN, M. R.; JENKINS, D. G.; KELLY, V.G. Resistance priming to enhance neuromuscular performance in sport: evidence, potential mechanisms and directions for future research. Sports Medicine, London, v.49, n. 10, p. 1499-1514, 2019.

HODGSON, M.; DOCHERTY, D.; ROBBINS, D. Post-activation potentiation motor performance. Sports Medicine, London, v. 35, n. 7, p. 585-95, 2005.

HOEGER, W. W. K.; HOPKINS, D. R.; BARETTE, S. L.; HALE, D. F. Relationship between repetitions and selected percentages of one repetition maximum: A comparison between untrained and trained males and females. Journal of Applied Sport Science Research, Lincoln, v. 4, n. 2, p. 47-54, 1990.

HOPKINS, W. G. Measures of reliability in sports medicine and science. Sports Medicine, London, v. 30, n. 1, p. 1-15, 2000.

JO, E.; JUDELSON, D. A.; BROWN, L. E.; COBURN, J. W.; DABBS, N. C. Influence of recovery duration after a potentiating stimulus on muscular power in recreationally trained individuals. Journal of Strength and Conditioning Research, Philadelphia, v. 24, n. 2, p. 343-7, 2010.

KHAMOUI, A. V.; BROWN, L. E.; COBURN, J. W.; JUDELSON, D. A.; URIBE, B. P.; NGUYEN, D.; TRAN, T.; EURICH, A. D.; NOFFAL, G. J. Effect of potentiating exercise volume on vertical jump parameters in recreationally trained men. Journal of Strength and Conditioning Research, Philadelphia, v. 23, n. 5, p. 1465-9, 2009.

KILDUFF, L. P.; BEVAN, H.; OWEN, N.; KINGSLEY, M. I. C.; BUNCE, P.; BENNETT, M.; CUNNINGHAM, D. Optimal loading for peak power output during the hang power clean in professional rugby players. International Journal of Sports Physiology and Performance, New Milford, v. 2, n. 3, p. 260-9, 2007.

LUSCHER, H. R.; RUENZEL, P.; HENNEMAN, E. Composite EPSPs in motoneurons of different sizes before and during PTP: Implications for transmission failure and its relief in la projections. Journal of Neurophysiology, Rockville, v. 49 , n. 1 , p. $269-89,1983$.

MAHLFELD, K.; FRANKE, J.; AWISZUS, F. Postcontraction changes of muscle architecture in human quadriceps muscle. Muscle and Nerve, New Jersey, v. 29, n. 4 , p. $597-600,2004$

MOORE, R. L.; STULL, J. T. Myosin light chain phosphorylation in fast and slow skeletal muscles in situ. American of Journal Physiology, Maryland, v. 5, n. 1, p. 462-71, 1984

MOURA, F. A.; JACINTO, C. A. C.; FELICISSIMO, C. T.; PRUDÊNCIO, M. V.; MERCADANTE, L. A.; CUNHA, S. A. Concordância e correlação entre três métodos distintos para a quantificação da altura do salto vertical. Revista Brasileira de Educação Física e Esporte, São Paulo, v. 29, n. 1, p. 25-34, 2015.

PÄÄSUKE, M.; SAAPAR, L.; ERELINE, J.; GAPEYEVA, H.; REQUENA, B.; ÖÖPIK, V. Postactivation potentiation of knee extensor muscles in power-and endurance-trained, and untrained women. European Journal of Applied Physiology, Berlim, v. 101, n. 5, p. 577-85, 2007

RASSIER, D. E.; MACINTOSH, B. R. Coexistence of potentiation and fatigue in skeletal muscle. Brazilian Journal of Medical and Biological Research, Rlbeirão Preto, v. 33, n. 5, p. 499-508, 2000

RHEA, M. R. Determining the magnitude of treatment effects in strength training research through the use of the effect size. Journal of Strength and Conditioning Research, Philadelphia, v. 18, n. 4, p. 918-20, 2004.

RIXON, K.; LAMONT, H.; BEMBEN, M. Influence of type of muscle contraction, gender, and lifting experience on postactivation potentiation performance. Journal of Strength and Conditioning Research, Philadelphia, v. 21 n. 2 , p. $500-5,2007$

ROBBINS, D. W.; DOCHERTY, D. Effect of loading on enhancement of power performance over three consecutive trials. Journal of Strength and Conditioning Research, Philadelphia, v. 19, n. 4, p. 898-902, 2005.

SALE, D. Postactivation potentiation: role in human performance. Exercise and Sport Sciences Reviews, Indianapolis, v. 30, n. 3, p. 138-43, 2002.

SMILIOS, I.; PILIANIDIS, T.; SOTIROPOULOS, K.; ANTONAKIS, M.; TOKMAKIDIS, S. P. Short-term effects of selected exercise and load in contrast training on vertical jump performance. Journal of Strength and Conditioning Research, Philadelphia, v. 19, n. 1, p. 135-9, 2005.

SZCZESNA, D.; ZHAO, J.; JONES, M.; ZHI, G.; STULL, J.; POTTER, J.D. Phosphorylation of the regulatory light chains of myosin affects $\mathrm{Ca} 2+$ sensitivity of skeletal muscle contraction. Journal of Applied Physiology, Washington, v. 92, n. 4 , p. $1661-70,2002$

TILLIN, N. A.; BISHOP, D. Factors modulating post-activation potentiation and its effect on performance of subsequent explosive activities. Sports Medicine, London, v. 39, n. 2, p. 147-66, 2009.

VANDENBOOM, R.; GRANGE, R. W.; HOUSTON, M. E. Myosin phosphorylation enhances rate of force development in fast-twitch skeletal muscle. American Journal of Physiology - Cell Physiology, Maryland, v. 268, n. 3 , p. C596-603, 1995.

YOUNG, W. B.; JENNER, A.; GRIFFITHS, K. Acute enhancement of power performance from heavy load squats. Journal of Strength and Conditioning Research, Philadelphia, v. 12, n. 2, p. 82-4, 1998.

\section{CONFLITO DE INTERESSE}

Os autores do estudo declaram não haver conflito de interesses.

\section{FINANCIAMENTO}

O autor Ramon Franco Carvalho recebe financiamento da Fundação de Amparo à Pesquisa do Estado do Rio de Janeiro (FAPERJ)

\section{ORCID E E-MAIL DOS AUTORES}

Danilo Jamaikon Carvalho

ORCID: 0000-0002-8101-5129.

E-mail: danilojcarvalho@yahoo.com.br

Heitor Francisco Moreira Barbosa

ORCID: 0000-0001-6822-3751.

E-mail: heitorbarbosa192010@hotmail.com

Ramon Franco Carvalho (Autor Correspondente)

ORCID: 0000-0003-3093-582X.

E-mail: prof.ramonfranco@gmail.com 